\title{
Otto von Bismarcks Leibarzt Ernst Schweninger und seine Rolle in der Dermatologie ${ }^{*}$
}

\author{
Otto von Bismarck's Personal Physician Ernst Schweninger and his \\ Importance in Dermatology
}

Autor

Institut

\author{
V. Wendt
}

Dermatologe, Westerstede
Bibliografie

DOI http://dx.doi.org/

10.1055/s-0041-107341

Akt Dermatol 2015; 41: 524-528

(c) Georg Thieme Verlag KG

Stuttgart · New York

ISSN 0340-2541

Korrespondenzadresse

Dr. med. Volker Wendt

Dermatologe

Am Flach 19

26655 Westerstede

dr.wendt@gmx.net

\section{Zusammenfassung \\ $\nabla$}

Den Namen Ernst Schweninger (1850-1924) assoziieren die meisten Dermatologen mit der nach ihm und seinem Assistenten Fausto BuzziCantone (1858-1907) benannten Variante der Anetodermie, bei der es ohne entzündlich-zelluläre Reaktion direkt zu Atrophie kommt. Einer breiten Öffentlichkeit im In- und Ausland wird Ernst Schweninger gegen Ende des 19. Jahrhun-

\section{Das Bismarck-Jahr 2015 $\nabla$}

Am 1.April 2015 wäre der erste deutsche Reichskanzler Otto von Bismarck 200 Jahre alt geworden. Bei der Lektüre der zahlreichen Publikationen aus Anlass dieses Gedenktages stößt der Leser über kurz oder lang auf den Namen seines Leibarztes Ernst Schweninger, der 1882 im Alter von 32 Jahren die Behandlung des Kanzlers übernahm und ihn bis zu seinem Tode am 30.7.1898 begleiten sollte ( $\bullet$ Abb. 1).

Bismarck beschrieb seinen Zustand im Jahre 1882 mit den Worten: „Ich stand am Rande eines Nerven- und Gesundheitsbankrottes“ [1]. Und über Schweninger: „Er ist überhaupt der einzige Mensch in meinem Leben gewesen, der Macht über mich gewonnen hat und dem ich nahezu unbedingten Gehorsam leiste“ [2]. Wer ist dieser Arzt, über den Bismarck zu einer für ihn so ungewöhnlichen Einschätzung kommt?

\section{Kindheit, Jugend und erste Universitäts- laufbahn \\ $\nabla$}

Ernst Schweninger wurde am 15.Juni 1850 als vierter Sohn des praktischen Arztes Dr. Franz

\footnotetext{
* Nach einem Vortrag anlässlich der 48.Tagung der Deutschen Dermatologischen Gesellschaft in Berlin am 29. April 2015.
}

derts als Leibarzt des Reichskanzlers Otto von Bismarck (1815-1898) bekannt. In dieser Lebensphase wird Schweninger endgültig $\mathrm{zu}$ einem „homo politicus“, dessen Arztsein einen direkten Einfluss auf den Gang der deutschen Geschichte nimmt. In einem zunehmenden Konflikt mit der sogenannten „Schulmedizin“ wird Schweninger darüber hinaus zu einem bedeutenden Wegbereiter der Naturheilkunde und der Psychosomatik.

Schweninger und seiner Ehefrau Fanny, geb. von Schacky, in Freystadt in der Oberpfalz geboren. Mit der Beförderung des Vaters zum Landgerichtsarzt verzog die Familie nach Neumarkt/ Oberpfalz. Alle vier Söhne besuchten das Gymnasium in Regensburg. Zum Wintersemester 1867/ 68 wurde Ernst Schweninger an der Universität München immatrikuliert. Dem eigentlichen Medizinstudium waren damals zunächst zwei Semester Philosophie vorangestellt. Noch während seines Studiums leistete er während des Deutsch-Französischen Krieges 1870/71 als „Nicht-Combattant“ freiwilligen Dienst in der Pflege Verwundeter auf den Schlachtfeldern. Bereits am 20.7.1872 promovierte Schweninger mit einer Arbeit „Über das Enchondrom“, 1873 legte er das Staatsexamen ab. Die Studienleistungen waren stets herausragend, ein „Sitten-Zeugniss“ der Ludwig-Maximilians-Universität München vom 8.1.1873 bescheinigte ihm zudem ein „klagefreies Betragen“. Dennoch bleibt festzuhalten, dass Schweninger in jenen Jahren einen eher hedonistischen Lebensstil pflegte [3] ( $\bullet$ Abb.2). Nur zwei Jahre nach dem Staatsexamen legte Schweninger als Assistent von Ludwig von Buhl am Pathologischen Institut der Universität München seine Habilitationsschrift „Über Transplantation und Implantation von Haaren“ vor [4]. Auch diese Arbeit wurde hoch gelobt und selbst im fernen Berlin von Rudolf Virchow (18211902) anerkennend gewürdigt. Niemand zweifel- 


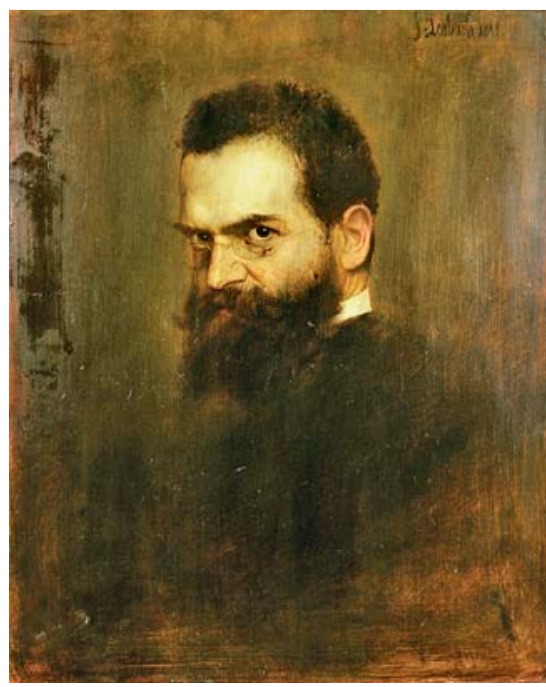

Abb. 1 Ernst Schweninger (1850-1924). Gemälde von Franz von Lenbach, 1888. Quelle: Archiv der Otto-vonBismarck-Stiftung Friedrichsruh. Standort: Bismarck-Museum Friedrichsruh.

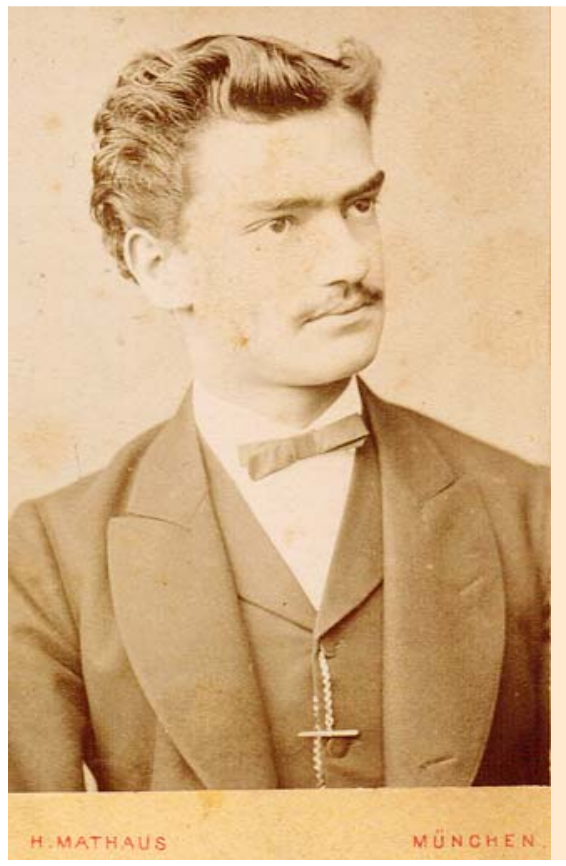

Abb. 2 Ernst Schweninger als junger Arzt um 1873. Quelle: Stadtmuseum Neumarkt.

te zu diesem Zeitpunkt an der sich abzeichnenden, glänzenden Universitätskarriere des noch jungen Mediziners. Der Privatdozent für pathologische Anatomie hielt ab dem Sommersemester 1876 regelmäßig Vorlesungen und trat in den Jahren 1875 bis 1879 mit 18 Publikationen in Erscheinung [3].

\section{Entlassung von der Universität und Haftstrafe \\ $\nabla$}

Anfang März 1879 wurde gegen Ernst Schweninger Anzeige wegen „Vergehens wider die Sittlichkeit, begangen an einem öffentlichen Orte“, erstattet. Was war geschehen? Schweninger betreute in jener Zeit die Ehefrau eines befreundeten Kollegen als Privatpatientin. Aus dieser Arzt-Patienten-Beziehung entstand eine Beziehung. Beide trafen sich wiederholt auf einem Friedhof. Anschuldigungen und Unschuldsbeteuerungen sind im Detail den Gerichtsakten zu entnehmen. Die Boulevard-Presse berichtete über die Gerichtsverhandlung und das abschließende Urteil, in welchem Ernst Schweninger vom königlichen Bezirksgericht München zu einer Gefängnisstrafe von vier Monaten ver- urteilt wurde. Schweninger ging zweimal in Berufung, ohne jedoch das Gericht von seiner Unschuld überzeugen zu können. Schließlich formulierte er am 12.11.1879 sein Gesuch um Enthebung von der Funktion eines Privatdozenten der Universität München, „so lange nicht der Beweis geliefert sein wird, daß ich mich dieser Ehre nicht unwürdig gemacht habe“ [3].

\section{Eine neue Existenz \\ $\nabla$}

Nach verbüßter Haft ging Schweninger vorübergehend nach Nizza und kehrte 1881 zurück nach München, um sich dort als praktischer Arzt niederzulassen. Innerhalb kürzester Zeit erlangte er den Ruf eines außerordentlich erfolgreichen Mediziners, dessen Bereitschaft, die Patienten in ihrer Individualität wahrzunehmen, für die damalige Zeit höchst ungewöhnlich war. $\mathrm{Zu}$ diesen Patienten gehörte auch Graf Wilhelm von Bismarck (1852-1901). Er war der älteste Sohn Otto von Bismarcks und hatte Schweninger im Herbst 1881 erstmals wegen einer Gichterkrankung konsultiert. Wilhelm von Bismarck war auch der Wegbereiter für die Einladung des jungen Arztes nach Varzin in Pommern, dem damaligen Sitz der Familie Bismarck. Anlass war die Schwere der Erkrankung seines Vaters [5].

\section{Der Patient Otto von Bismarck}

Bei ihrer ersten Begegnung am 4.Oktober 1882 fand Schweninger den Reichskanzler in einem desolaten Gesundheitszustand vor. Der mit $124 \mathrm{~kg}$ stark übergewichtige Bismarck litt u.a. an chronischer Schlaflosigkeit, Migräne, einer Trigeminusneuralgie sowie „Magencrisen“. Zudem war von dem wohl berühmtesten deutschen Internisten der damaligen Zeit, Theodor von Frerichs (1819-1885), ein Leberkarzinom diagnostiziert worden. Es wurde Bismarck eine verbliebene Lebenszeit von ca. 3 Monaten eingeräumt [6, 7,8]. Das Essverhalten des Reichskanzlers lief allen ärztlichen Bemühungen völlig zuwider: Roastbeef und Beefsteak zum Frühstück, weitere Wildgerichte und Geflügel, danach Pudding, Bier, Rotwein und Champagner, mittags erneut ein schweres Essen mit sechs Gängen, gegen Mitternacht Tee [9]. Den früheren Ärzten stand Bismarck in tiefem Misstrauen gegenüber, von einem vertrauensvollen Verhältnis konnte bis zur Ankunft Schweningers überhaupt nicht die Rede sein.

Schweninger erhob zunächst eine ausführliche Anamnese und soll den zunehmend ungeduldig werdenden Kanzler mit den Worten diszipliniert haben, „sich doch an einen Viehdoktor zu wenden, wenn er nicht reden wolle“. In späteren Ausführungen wurde dieser Akt der „Insubordination“ von Schweninger immer wieder in das Reich der Legenden verwiesen [10]. Schweninger forderte von Bismarck „ein rückhaltloses Vertrauen und den grundsätzlichen Ausschluß jeglicher Einflüsse von dritter Seite“. Das kompromisslose Auftreten des jungen Arztes, der über eine außergewöhnliche Suggestivkraft verfügte, muss dem Kanzler letztendlich gefallen haben. Bismarck fügte sich in sein Schicksal. Er stand fortan unter der strengen Aufsicht seines Arztes Dr. Schweninger, dessen Hauptaugenmerk zunächst der Lebensführung Bismarcks galt. Scherzhaft bezeichnete dieser Schweninger auch gerne als seinen „schwarzen Tyrannen“ [11]. Für Bismarck war die von Schweninger veranlasste „Rückkehr zur Natur“ der Schlüssel zu seiner Genesung. Beispielhaft ist folgende Begebenheit: In einer der schlaflosen Nächte soll sich Schweninger an Bismarcks Bett gesetzt und seine Hand genommen haben „wie die 


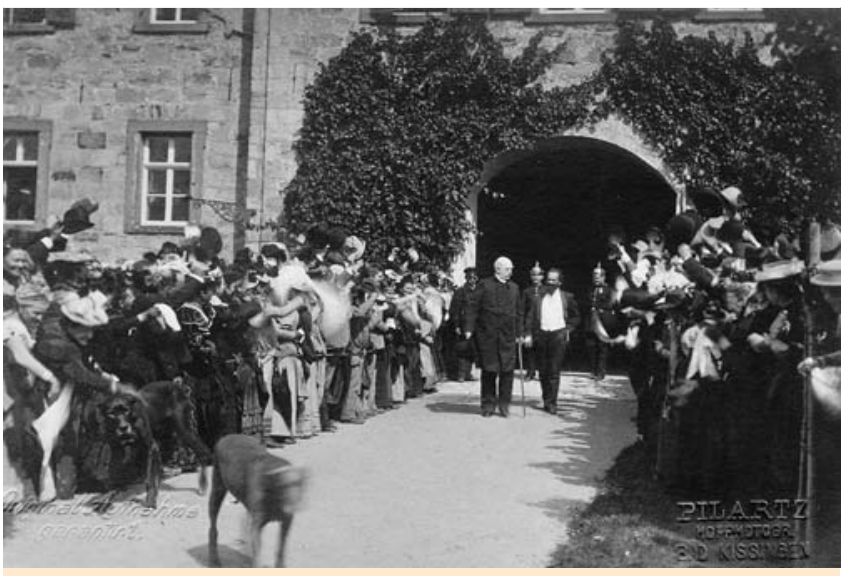

Abb.3 Otto von Bismarck und Ernst Schweninger vor der Oberen Saline in Bad Kissingen um 1890. Quelle: Stadtarchiv Bad Kissingen.

Mutter bei einem unruhigen Kind“. Am nächsten Morgen - Bismarck hatte durchgeschlafen - habe der Arzt noch immer an derselben Stelle gesessen [9]. Nachdem sich das zuvor von anderer Seite diagnostizierte Leberkarzinom als Gallenstein herausstellte und die Gesundung Bismarcks weiter voranschritt, sorgte sich dieser um die Zukunftspläne Schweningers. Er schrieb: „Ohne Schweninger kann ich nicht gesund leben. Wenn er weggeht, muss ich nach München ziehen“ [12]. Für den in seinen Grundüberzeugungen so preußisch geprägten Reichskanzler war das geradezu unvorstellbar. So wurde an einer alternativen Lösung

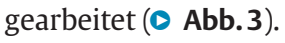

\section{Antritt einer zweiten Universitätslaufbahn \\ $\nabla$}

Auf Betreiben des Kanzlers wird Schweninger im Februar 1884 der Professorentitel verliehen und am 31.7.1884 erfolgte seine Bestallung zum a.o. Professor in der medizinischen Fakultät der Universität Berlin. Mit der Bestallung war das Extraordinariat für Dermatologie verbunden. Die Hautklinik war zuvor von Georg Richard Lewin (1820-1896) geführt worden. Dieser musste die Abteilung für Hautkrankheiten an Schweninger abtreten und behielt die Leitung der Syphilisklinik [13]. Der Widerstand der Fakultät gegen Schweninger als Dermatologie-Professor „von Bismarcks Gnaden“ war erbittert. Unter der Führung Rudolf Virchows erreichte die erregte Debatte schließlich im Februar 1885 sogar das preußische Abgeordnetenhaus. Virchow, zu diesem Zeitpunkt Abgeordneter der liberalen „Deutschen Freisinnigen Partei“ und entschiedener Gegner Bismarcks, führte insbesondere die Verurteilung Schweningers aus dem Jahr 1879 an, um den Akt der „Erniedrigung der deutschen Fakultäten und Universitäten“ anzuprangern. Er sah sogar die Möglichkeit einer „sittlichen Gefährdung der Medizinstudenten“ [14]. So waren die Voraussetzungen für eine gedeihliche Zusammenarbeit bei Amtsantritt denkbar ungünstig. Der Physiologe Emil du Bois-Reymond (1818-1896) lehnte sogar einen Antrittsbesuch Schweningers in seinem Institut ab, woraufhin dieser sich mit du Bois-Reymond duellieren wollte. Zur Austragung dieser Ehrenstreitigkeit ist es nie gekommen $[7,15]$. Die satirische Wiener Wochenschrift „Der Floh“ karikierte in ihrer Ausgabe vom 17. August 1884 Schweninger auf ihrem Titelblatt als „Protectionskind“, das sich nicht rühmen kann, die Leibes- oder Geistesfrucht der Berliner Alma mater zu sein [16]. Zu den wenigen Wissenschaftlern, die Schweninger

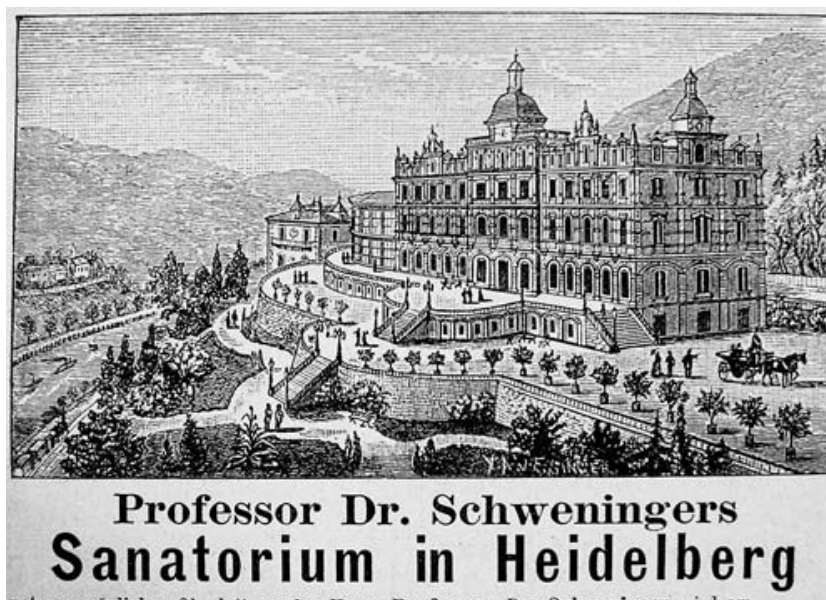

\section{anter persónlicher Oberleitung des Herrn Professors Dr. Sclıweninger wird am} 15. März 1887

fir alle Formen chronischer Cirkulations- und Ernïhrungs-Störungen dauernd eröffne Aerztliche Anfragen beliebe man nach Berlin an Hern Professor Dr. Schwening
merstrasse 100, zu richten; alle anderen Luskūnfte werden vom Sanatorium in Heidel

Heidelberg, den 16. Februar 1887.

Die Direktion.

Abb.4 Zeitungsinserat zur Eröffnung des Schweninger-Sanatoriums in Heidelberg am 15.3.1887. Quelle: Archiv der Otto-von-Bismarck-Stiftung Friedrichsruh.

an der Charité vorurteilsfrei aufnahmen und mit ihm interkollegial zusammenarbeiteten, gehörte Robert Koch (1843-1910).

Schweninger beschrieb die Verhältnisse in der Berliner Hautklinik bei seinem Amtsantritt als „kümmerlich“. Die Abteilung bestand aus fünf kleinen, dunklen Zimmern mit niedriger Deckenhöhe im dritten Stockwerk der neuen Charité. Zusätzlich verfügte er über ein weiteres Zimmer in einem Nebengebäude im Garten. Insgesamt konnten damit 40 Patienten untergebracht werden. Trotz dieser schwierigen Startbedingungen stürzte sich Schweninger förmlich in seine neuen Aufgaben an der Berliner Universität, um „im Sinne unserer Vorgänger ehrlich und gewissenhaft für Unterricht und Wissenschaft weiterzuwirken“ [3]. Aus der Rückschau bleibt jedoch festzuhalten, dass lediglich die 1891 von Schweninger und seinem Assistenzarzt Fausto Buzzi-Cantone beschriebene Variante der Anetodermie, bei der es ohne entzündlich-zelluläre Reaktion direkt zur Atrophie kommt, als bleibender Beitrag auf dermatologischem Fachgebiet gewertet werden kann [17]. Mit der in jener Zeit heranreifenden Erkenntnis, wissenschaftlich nur wenig erreichen zu können, wandte sich Schweninger bereits 1886/87 zusätzlichen neuen Betätigungsfeldern zu und gründete „Professor Dr. Schweningers Sanatorium Schloß Heidelberg“ ( $\bullet$ Abb.4).

\section{Neue und alte Aufgaben außerhalb der Universität, Bismarcks Tod \\ $\nabla$}

Für Schweninger standen die Körperkonstitution und ihre Beeinflussung durch physikalische Heilmethoden im Zentrum seiner wissenschaftlichen Überzeugung. Diese für jene Zeit unkonventionellen Therapieansätze wurden von Schweninger in seinem Heidelberger Sanatorium erstmals in einem größeren Umfang erfolgreich erprobt, wobei die „Ätiologie-Directiven“ Schweningers für jeden einzelnen Patienten individuell ausgearbeitet wurden ( $\bullet$ Abb.5). 1890 wurde das Sanatorium wieder geschlossen. Schweninger hatte sich zeitlich und finanziell übernommen. Betriebswirtschaftliche Überlegungen waren ihm völlig fremd. Neben seiner Tätigkeit an der Berliner Charité galt seine Haupt- 

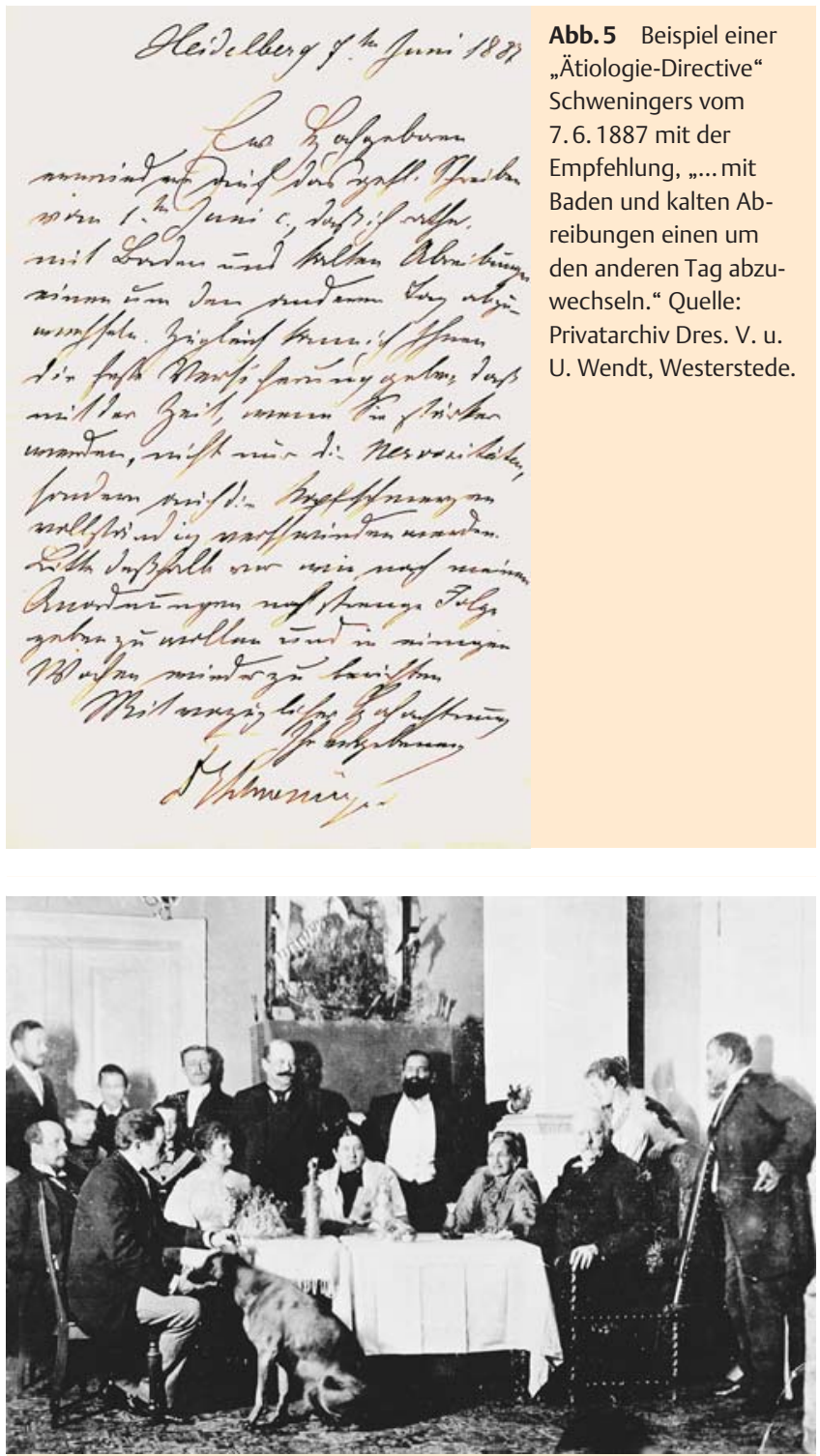

Abb.6 Otto von Bismarcks Entourage in Friedrichsruh. Ernst Schweninger am Kachelofen stehend. Am linken äußeren Bildrand Rudolph Chrysander. Quelle: Stadtmuseum Neumarkt.

sorge unverändert dem Reichskanzler Bismarck, der in seinen letzten Lebensjahren in seinem Schloss Friedrichsruh im Sachsenwalde östlich von Hamburg lebte. Obwohl unmittelbar an der Bahnstrecke Berlin-Hamburg gelegen, konnte Schweninger unmöglich im Hause Bismarck omnipräsent sein. So wirkte der junge Dr. Rudolph Chysander (1865-1950) als Assistent Schweningers in Dauerstellung vor Ort in Friedrichsruh. Beide gehörten neben dem Maler Franz von Lenbach (1836-1904) und seiner Frau zur engsten Entourage der Familie Bismarck ( $\bullet$ Abb.6).

Am 30.Juli 1898 starb Otto von Bismarck im Alter von 83 Jahren an den Folgen eines akuten Lungenödems in Friedrichsruh. Über 17 Jahre hatte Ernst Schweninger seinen prominenten Patienten begleitet. Die im Jahre 1882 von anderer Seite gestellte Diagnose eines Leberkarzinoms erwies sich als nicht richtig. Dank der von Schweninger eingeleiteten, radikalen Veränderung seiner Lebensführung blieb Bismarck bis kurz vor seinem Tod arbeitsfähig. In diese Zeit fielen wichtige Konferenzen und Abkommen, die der Friedenssicherung in einem maßgeblich von Bismarck geprägten Bündnissystem dienten [5]. Auch nach der Entlassung Bismarcks

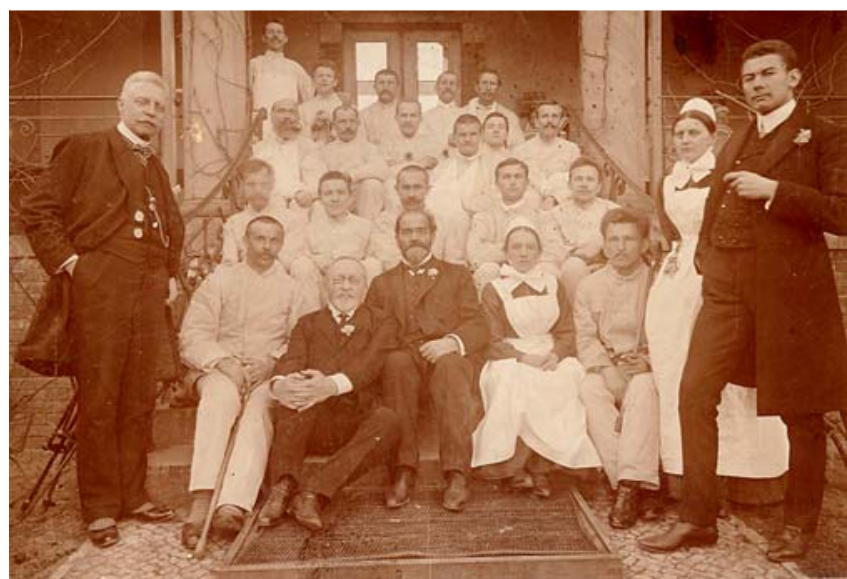

Abb.7 Ernst Schweninger (1. Reihe, Bildmitte) mit seinen Mitarbeitern im Kreiskrankenhaus Groß-Lichterfelde bei Berlin um 1902. Quelle: Stadtmuseum Neumarkt.

im Jahre 1890 war seine Autorität als „elder statesman“ immer noch von großer innen- und außenpolitischer Bedeutung. Schweninger wurde somit zu einem „homo politicus“, dessen Arztsein einen direkten Einfluss auf den Gang der deutschen Geschichte nahm.

\section{Privates Glück, Gründung einer Ärzteschule \\ $\nabla$}

Am 16.6.1898 heirateten Ernst Schweninger und Maria Magdalena, geschiedene von Lenbach, geborene Reichsgräfin von Moltke, auf der Nordseeinsel Helgoland. Aus der Ehe sollten vier Kinder hervorgehen.

Schweninger verfolgte um die Jahrhundertwende mit Nachdruck die Gründung einer eigenen Ärzteschule. Nach seiner klinischen Arbeit war Schweninger überzeugt vom Grundgedanken, dass Ärzte menschlich fühlen müssen und human ihre Aufgabe empfinden sollen. Sie müssten die einengenden „Scheuklappen der Wissenschaft“ abzulegen bereit sein. Schweninger wollte mit seiner Ärzteschule und dem Leitbild des Arztes als „humaner Künstler“ eine Reform der Medizin einleiten. Zunächst hielt er regelmäßige Kolloquien in seiner Berliner Wohnung. Mit der Ernennung Schweningers zum Ärztlichen Direktor des neuen Kreiskrankenhauses in Groß-Lichterfelde bei Berlin im Jahre 1900 waren die Voraussetzungen für einen weiteren Ausbau seiner Ärzteschule geschaffen ( Abb.7). Das Krankenhaus GroßLichterfelde galt als das erste deutsche Modell-Krankenhaus für Naturheilkunde. Pro Jahr kamen mehr als 300 Ärzte, um an den Kolloquien und Patientenvorstellungen im Krankenhaus teilzunehmen [15]. Als Publikationsorgan diente das „Archiv für physikalisch-diätetische Therapie“. Zu den bekanntesten Schülern zählte Georg Groddeck (1866-1934), ein Wegbereiter der Psychosomatik. Groddeck war bereits als Assistenzarzt in der Hautklinik der Charité zusammen mit Schweninger tätig und bezog sich in seinen zahlreichen Publikationen immer wieder auf die in der Hautklinik und der Ärzteschule Ernst Schweningers gesammelten Erfahrungen [18,19].

Mit seinem 1906 erschienenen Buch „Der Arzt“ ist Schweninger eine Typologie des Arztes gelungen, die seine Gedanken über den ärztlichen Beruf und die Stellung des Arztes in der Gesellschaft auf 148 auch heute noch lesenswerten Seiten darstellt [20]. Die naturheilkundliche Ausrichtung spiegelte sich auch in seinen 
Therapieprinzipien wider. Schweninger wollte keine „Fälle“ behandeln, sondern immer den ganzen Menschen. Das oberste Gebot war das Individualisieren und der Kampf gegen die „Schablone“. Physikalische und diätetische Behandlungen standen dabei ganz im Vordergrund.

\section{Ein neuer Lehrauftrag und endgültiger Abschied von der Universität \\ $\nabla$}

Im Juli 1902 gab Schweninger die Leitung der Dermatologischen Klinik an der Charité auf. Mit dem Tode Bismarcks hatte er seinen mächtigen Fürsprecher verloren, der über viele Jahre schwelende Konflikt mit der Schulmedizin hatte sich durch die Gründung seiner Ärzteschule weiter intensiviert. Schweninger erhielt als Kompensation die Leitung einer Poliklinik für Innere Krankheiten an der Charité sowie einen neuen Lehrauftrag für Geschichte der Medizin und für Allgemeine Pathologie und Therapie, den er bis 1906 innehatte [13,21]. Mit der Übersiedlung nach Pullach bei München im gleichen Jahr endeten Schweningers zweite Universitätslaufbahn und auch die Leitung des Kreiskrankenhauses in Groß-Lichterfelde.

Bereits im Jahre 1900 erlitt Schweninger einen folgenschweren Unfall, von dem er sich bis zu seinem Tode nicht mehr richtig erholen sollte. Der Unfall ereignete sich während einer Nordlandschiffsreise mit Friedrich Alfred Krupp. Im Rahmen einer gemeinschaftlichen Turnübung auf dem vereisten Deck glitt Schweninger aus und stürzte einen eisernen Abgang hinunter in den Maschinenraum. Die Folge war ein komplizierter Beckenbruch mit zahlreichen Knochensequestern, die Schweninger nicht operativ revidieren ließ, da er von der Heilkraft des Körpers überzeugt war und chirurgischen Maßnahmen stets misstrauisch gegenüberstand [5].

Am 13.1.1924 starb Ernst Schweninger im 74. Lebensjahr an den Folgen eines apoplektischen Insultes. Die Beerdigung fand auf dem Friedhof in München-Solln statt. Die Grabstelle ist bis zum heutigen Tage erhalten.

Bei der Betrachtung seiner Lebensstationen wird deutlich, dass man Ernst Schweninger nicht gerecht wird, wenn man ihn auf seine Funktion als Leibarzt Otto von Bismarcks reduziert. Schweninger sah sich selbst als Reformator des Arzttums. Betrachtet man die für damalige Verhältnisse ungewöhnlichen Heilmethoden, so erkennt man in ihm einen bedeutenden Wegbereiter sowohl der Naturheilkunde als auch der Psychosomatik, der seiner Zeit in vielem voraus war.

\section{Danksagung}

$\nabla$

Der Autor bedankt sich beim Bundesarchiv in Berlin, der Ottovon-Bismarck-Stiftung in Friedrichsruh sowie dem Stadtarchiv Bad Kissingen, die durch ihre unkomplizierte Kooperationsbereitschaft vor allem die Illustration der Arbeit ermöglicht haben. Ein besonderer Dank gilt der Leiterin des Stadtmuseums Neumarkt, Frau Petra Henseler, für ihren besonderen Einsatz bei der Bereitstellung ihrer Archivmaterialien.

\section{Interessenkonflikt}

$\nabla$

Der Autor gibt an, dass kein Interessenkonflikt besteht.

Abstract

\section{Otto von Bismarck's Personal Physician Ernst Schweninger and his Importance in Dermatology \\ $\nabla$}

The name Ernst Schweninger (1850 - 1924) will be associated by most of the dermatologists with the variant of anetoderma reported as the Schweninger-Buzzi type, named after him and his assistant Fausto Buzzi-Cantone (1858 - 1907). The SchweningerBuzzi type is noninflammatory from the beginning. At the end of the 19th century Ernst Schweninger becomes world-famous in the broad public as personal physician of the German chancellor Otto von Bismarck (1815-1898). In this period of life Schweninger turns out to be a "homo politicus“, whose profession exerts direct influence on the course of German history. In an increasing conflict with the so-called „school-medicine“ Schweninger becomes in addition an important precursor of naturopathy and psychosomatic medicine.

\section{Literatur}

1 Fürst von Bismarck O. Gedanken und Erinnerungen. Stuttgart, Berlin: Cotta'sche Buchhandlung; 1928

2 Thies J. Die Bismarcks. Eine deutsche Dynastie. München: Piper; 2013

3 Espach A. Beiträge zur Biographie Ernst Schweningers. München: Vereinigung für Geschichte der Medizin; 1979

4 Schweninger E. Über Transplantation und Implantation von Haaren. Z Biol 1875; 11: $341-380$

5 Schwarz G. Ernst Schweninger. Leipzig: Reclam; 1941

6 Schweninger E. Dem Andenken Bismarcks. Leipzig: Hirzel; 1899

7 Quecke K. Ernst Schweninger, der Leibarzt Bismarcks. Med Welt 1951; 20: $500-505$

8 Ziegelroth P. Dem Andenken Schweningers. Westermanns Monatshefte 1924; 68: 295-297

9 Thies J. Der Herr im Haus. Zeit Geschichte 2014; 4: 78-82

10 Krecke A. Vom Arzt und seinen Kranken. München: J F Lehmanns; 1932

11 Zehmisch H. Der kranke Reichskanzler Otto v. Bismarck und sein Leibarzt Dr. Ernst Schweninger. Ärzteblatt Sachsen 2003; 11: 495-497

12 Kleine HO. Der schwarze Tyrann; Zur Erinnerung an Ernst Schweninger, Bismarcks Leibarzt. Dtsch med Wschr 1938; 64: 1739-1740

13 Klaschka F, Rauhut K, Hrsg. 100 Jahre Dermatologie in Berlin. Berlin: Grosse; 1984

14 Scholz A. Geschichte der Dermatologie in Deutschland. Berlin: Springer; 1999

15 Brauchle A. Geschichte der Naturheilkunde in Lebensbildern. Stuttgart: Reclam; 1951

16 Anonymus X. Bismarck's Leibarzt. Der Floh 1884; 33: 1-2

17 Schweninger E, Buzzi F. Multple benign tumourlike new growths of the skin. In: Unna PG, Morris M, Besner E et al., eds. International atlas of rare skin diseases. Hamburg: Voss; 1891: 4-5

18 Groddeck G. Ernst Schweninger. Der Arzt; Zschr f wissensch Naturheillehre u Naturheilkunst 1930; 2: 167-174

19 Groddeck G. Psychoanalytische Schriften zur Psychosomatik. Wiesbaden: Limes; 1966

20 Schweninger E. Der Arzt. Radeburg: Verlag Dr. Madaus; 1926

21 Klein E. Bericht über die ärztliche Tätigkeit an der Poliklinik für innerlich Kranke für die Etatsjahre 1902 1906. Char A Berlin 1907; 31: 645 733 